\title{
IMPACT OF GENERATIONAL COMMONALITY OF SHORT- LIFE CYCLE PRODUCTS IN MANUFACTURING AND REMANUFACTURING PROCESSES
}

\author{
Kim, Jinju; Kim, Harrison \\ University of Illinois at Urbana-Champaign
}

\begin{abstract}
Short-life cycle products are frequently replaced and discarded despite being resource-intensive. The short life span and the low utilization rate of the end-of-life products cause severe environmental problems and waste of resources. In the case of short-life cycle products, a new generation of products is released sooner than other products, therefore there are the opportunities to have various generations of products during the remanufacturing process. The commonality between generations increases the intergenerational component compatibility, which increases the efficiency of the manufacturing and remanufacturing processes, while at the same time weakening the performance difference between generations. This paper proposes a mathematical model to investigate the effect of commonality among generations on the overall production process. Based on various given new generation product designs with different commonality, we aim to propose optimal production planning and pricing strategies to maximize the total profitability and investigate how the results vary according to the commonality strategies between product generations.
\end{abstract}

Keywords: Sustainability, Design for X (DfX), Ecodesign

Contact:

Kim, Harrison

University of Illinois at Urbana-Champaign

Industrial and Enterprise Systems Engineering

United States of America

hmkim@uiuc.edu

Cite this article: Kim, J., Kim, H. (2019) 'Impact of Generational Commonality of Short-Life Cycle Products in Manufacturing and Remanufacturing Processes', in Proceedings of the 22nd International Conference on Engineering Design (ICED19), Delft, The Netherlands, 5-8 August 2019. DOI:10.1017/dsi.2019.340 


\section{INTRODUCTION}

There is no doubt that advances in electronic technology have revolutionized the world and people's daily lives. Behind this convenience, however, is the enormous amount of natural resources and greenhouse gas emissions for short-term use. Moreover, the post-use products become electronic waste, which creates various problems (Greenpeace, 2017). As these problems become more serious, global awareness of the environment and environmental regulations have been strengthened (Kwak et al., 2009; Kim and Moon, 2017). These changes have forced manufacturers and product designers to develop ecodesign of their products with an emphasis on the entire product life cycle (Aydin et al., 2015; Kwak and Kim, 2017; Kim and Moon, 2017).

One of the effective ways to improve product sustainability is recovering end-of-life (EoL) products after customer use, such as recycling, remanufacturing/refurbishment, reuse, and reconditioning. Remanufacturing defines the process of using EoL products and returned products to restore them functionally and physically to their original or even better condition. Remanufacturing offers many advantages not only in economic but also in social aspects. From an economic point of view, it can help to reduce utilization of natural resources, shorten the production process, and provide opportunities to enter price-sensitive markets (Ijomah, 2002; Hatcher et al., 2011; Kwak and Kim, 2017). On the social side, it not only enhances the green brand image of enterprises, but also addresses relevant manufacturing environmental laws (Aydin et al., 2017).

Unlike products that are used for long periods of time, there are several issues that should be considered in the process of remanufacturing for products with short-life cycle. First, the effects of generational commonality should be considered. For short-life cycle products, new generations of products are faster to enter the market than other products. Therefore, different generations of products are often in the market at the same time. As a result, there is a high possibility that different generations of products will be introduced in the process of collecting EoL products (Kwak and Kim, 2011). The reusability of components from different generations is determined by the interaction of the design of individual products with the design of each generation. In other words, generational commonality can help increase the reuse rate of EoL components extracted from different generations.

However, technological obsolescence of previous generations should also be considered. For electronics with short-life cycle, rapid technological advances make more advanced components available quickly, leading to technological obsolescence of previous components. Therefore, it is less likely that components used in the previous generation from past years will meet the needs of customers in the market (Kwak, 2018). Differentiating quality, performance and appearance from previous generations is required to meet customer requirements and increase sales.

In order to deal with these issues, original equipment manufacturers (OEMs) with manufacturing and remanufacturing should consider the way generational commonality affects overall production process for a line of new and remanufactured product. How OEMs make design changes between product generations can have a significant impact on economic success and customer satisfaction. However, it is difficult to estimate the uncertainty within remanufacturing process and the satisfaction of the customer for a certain design of the product. There is also a lack of models to assess commonality between generations of products that consider both manufacturing and remanufacturing processes.

In previous papers, the effects of product commonality have been studied for advantages and disadvantages in terms of manufacturing and marketing traditionally. The commonality among products can increase the efficiency of the manufacturing process at low cost while satisfying various customer requirements. But at the same time, it will undermine the product's diversity and limit its performance (Simpson et al., 2001; Thevenot and Simpson, 2007). The commonality may have other significant consequences when considering product recovery strategies after the end of the product's life.

Kwak and Kim (2011) propose a study on the impact of sharing parts between product variants in the product family on profitability. The results show that high sharing product family design can generate high profits at the end-of-life stages. However, this paper only consider the portion of the product life cycle, such as end-of-life stage, rather than entire life cycle.

Other studies that consider remanufacturing and manufacturing simultaneously focus on a single product within one product generation (Aydin et al., 2015 Kwak and Kim, 2017). Kwak and Kim (2017) propose an integrated model that includes the buyback, production, and marketing processes for a line 


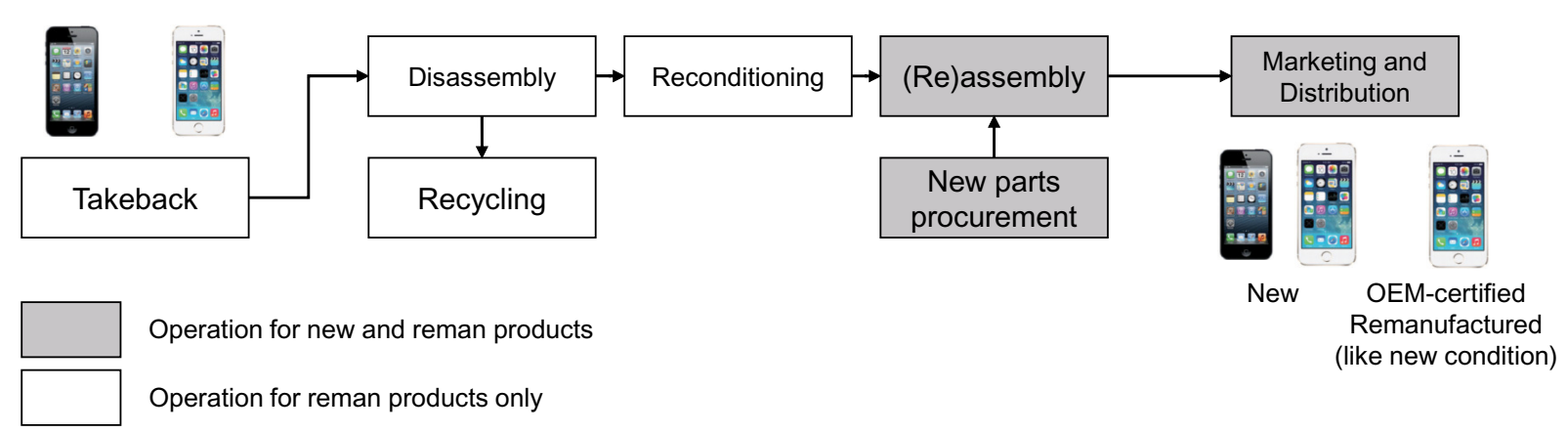

Figure 1. Production process under consideration (derived from Kwak \& Kim (2017))

of new and remanufactured products. This model assumes that the remanufactured product is of the same design as the EoL product and is not considering intergenerational component compatibility.

Kim and Moon (2017) propose the methodology to identify sustainable product family configuration by integrating sustainability performance and a platform strategy. Although this paper considers several products in the product family, it does not consider multi-generation. Also, this paper does not consider the detailed process required for remanufacturing.

This paper investigates the effect of generational commonality on remanufacturing and manufacturing process for products with short-life cycle. Specifically, the study aims to identify not only the way generational commonality affects production plans and pricing policies, but also the effectiveness of the remanufacturing process, such as the reuse rate of previous generation products. These results can provide insight into the product generational platform design and marketing strategies that consider both manufacturing and remanufacturing.

\section{PROPOSED MODEL}

\subsection{Problem statement}

This paper proposes a methodology to assess how the generational commonality of short-life cycle products affects the entire production processes. This paper finds the results that the determination of generational commonality can be represented in economic and environmental aspects. The objective function of mathematical models is to maximize profits while meeting environmental impact saving determined from environmental regulation or company objectives. For this purpose, this paper proposes an expanded model of the model used in the previous study (Kwak and Kim, 2017).

This model is intended for OEMs that manufacture and remanufacture short-life cycle products such as electronics. Remanufactured products are made with the same performance and appearance as the released models but are priced at lower prices due to customers who perceive them to be made from used product. Figure 1 shows the production process considered in this model.

In this study, the remanufacturing process includes the collection of EoL products, disassembly, conditioning, and reassembly. The collecting process for remanufacturing involves a wide range of generations, from previously released products to recently released products. OEMs can produce remanufactured products of specific models using various EoL and returned products. When EoL products are collected, the products are broken down into parts. It is assumed that the parts are simply recycled if they are not used in the remanufactured product model.

Conversely, if the parts work well and are used in the remanufactured production, they are reconditioned, reassembled and made into the remanufactured product. If parts are insufficient during the remanufacturing process, new parts can be acquired and used. New products are made using only new parts.

\subsection{Transition matrix for remanufacturing}

In this model, the various production processes (e.g., collecting, disassembly, recycling, reconditioning, reassembly) are modeled during the remanufacturing process using the transition matrix (Kwak and Kim, 2017) which was used in the previous paper. According to Kwak and Kim (2017), this transition matrix can handle various products at once, so it can address the issue of component commonality of different generations of products. 


\begin{tabular}{|c|c|c|c|c|c|c|c|c|c|c|}
\hline & \multirow{2}{*}{\multicolumn{2}{|c|}{ Operation }} & \multicolumn{3}{|c|}{ Disassembly } & & \multicolumn{3}{|c|}{ Reconditioning } & Reassembly \\
\hline & & & 1 & 2 & 3 & 4 & 5 & 6 & 7 & 8 \\
\hline \multirow[t]{2}{*}{ Remanufactured product } & $\overline{1}$ & Product ADE \& & & & & & & & & $\overline{1}$ \\
\hline & 2 & Product ABC (EOL 1) & -1 & & & & & & & \\
\hline \multirow{9}{*}{ End-of-life products } & 3 & Product ABC (EOL 2) & & -1 & & & & & & \\
\hline & 4 & Product ADE (EOL 1) & & & -1 & & & & & \\
\hline & 5 & Product ADE (EOL 2) & & & & -1 & & & & \\
\hline & 6 & Component $A \circledast$ & & & & & 1 & & & -1 \\
\hline & 7 & Component $D ®$ & & & & & & 1 & & -1 \\
\hline & 8 & Component $\mathrm{E} \otimes$ & & & & & & & 1 & -1 \\
\hline & & Component $\mathrm{A}(\mathrm{W})$ & 1 & 0.6 & 1 & 0.6 & -1 & & & \\
\hline & & Component B (W) & 1 & 0.7 & & & & & & \\
\hline & & Component $\mathrm{C}(\mathrm{W})$ & 1 & 0.3 & & & & & & \\
\hline \multirow[t]{7}{*}{ Components } & & Component $D(W)$ & & & 1 & 0.8 & & -1 & & \\
\hline & & Component $\mathrm{E}$ (W) & & & 1 & 0.4 & & & -1 & \\
\hline & & Component $\mathrm{A}(\mathrm{N})$ & & 0.4 & & 0.4 & & & & \\
\hline & & Component B (N) & & 0.3 & & & & & & \\
\hline & & Component $\mathrm{C}(\mathrm{N})$ & & 0.7 & & & & & & \\
\hline & & Component $\mathrm{D}(\mathrm{N})$ & & & & 0.2 & & & & \\
\hline & 18 & Component $\mathrm{E}(\mathrm{N})$ & & & & 0.6 & & & & \\
\hline
\end{tabular}

Figure 2. Transition matrix for remanufacturing

Figure 2 illustrates the conceptual transformation matrix used in this study. It includes several generations of products in one matrix to explore the component commonality relationship between generations. Basically, the transition matrix shows the input/output material flow. The columns indicate the remanufacturing operation of products. The value of each cell appears as one of $-1,0,1$. A value of -1 in each cell $(i, j)$ means that the product or part corresponding to $i$ enter the operation $j$. The value 1 means that the product or part corresponding to $i$ is produced by the operation $j$. A value of zero means that there is no relationship between row and column.

As an example of the expanded transition matrix, Figure 2 shows that $\mathrm{ABC}$, the product of the previous generation, enters the process and produces parts $\mathrm{A}, \mathrm{B}$ and $\mathrm{C}$ through disassembly. The number of available parts depends on the quality of the end-of-life product, and in this paper the quality of the product is divided into 'good' (EOL 1) and 'poor' (EOL 2) conditions. The next generation product, ADF, are broken down into A, D, and F through its third and fourth operations. If the products of these two generations are used to produce ADF remanufactured products of the next generation, part A extracted from $\mathrm{ABC}$ can be used in the remanufacturing process of $\mathrm{ADF}$ because part $\mathrm{A}$ is shared between the two generations.

\subsection{Mathematical model}

This subsection presents the mathematical model used in this study. Equation 1 represents an objective function, which means maximizing the profits of the manufacturing and remanufacturing. Profits from new products mean that the associated costs are excluded from the revenue from the new products. Profits from remanufactured products are calculated differently from those of new products because they go through some different processes than new ones. The cost of remanufacturing consists of recycling, takeback, operation, marketing, procurement and marketing cost.

Equation 2 represents inequality constraints. The constraint $g 1$ means that the number of products that can be taken back depends on the number of EoL products available in the market and the buyback price. The constraints $g 2$ and $g 3$ are production quantity constraints, which mean that the amount of production cannot be more than the demand by the market. The constraint $g 4$ indicates that the production quantity of the remanufactured product cannot be greater than the quantity of the collected EoL products. The constraint g5 indicates whether the reduced environment impact through remanufacturing meets the $\operatorname{target} \delta$. The environmental impact savings can be calculated by comparing the amount of environmental impact in remanufacturing to the amount expected to occur when a new product is produced in the same quantity.

Equation 3 represents equality constraints. These equality constraints serve to balance the input-output material flow of the transition matrix, which can be used to estimate the cost of re-manufacturing. The constraint $h 1$ indicates that products that are collected at the end of their life are recycled or put into the process of remanufacturing. For externally available parts, The constraint $h 2$ indicates that the number 
of parts required for reproduction is available from the outside. The constraint $h 3$ ensures the flow balance of parts that are not allowed to purchase external parts. The constraint $h 4$ indicates that $Z^{r}$ shall be produced in the final production process. The constraint $h 5$ defines that only parts that are available for external purchase can be procured external parts. h6 limits the unrealistic conditions under which the final remanufactured product is used for recycling. Equation 4 means variable non-negativity.

$$
\max \sum_{i \in I}\left(P_{i}^{n}-C_{i}^{n}\right) Z_{i}^{n}+P^{r} Z^{r}-\left(\sum_{j \in J} C_{j}^{M} M_{j}+\sum_{i \in I} \sum_{k \in K} P_{i}^{k} X_{i}^{k}+\sum_{l \in L} c_{l} Y_{l}+\sum_{j \in J} c_{j}^{N} N_{j}+c_{d} Z^{r}\right)
$$

with respect to $X_{i}^{k}, Y_{j}, Z_{i}^{n}, Z^{r}, M_{j}, N_{j}, P_{i}^{n}, P^{r}, P_{i}^{k}(\forall i \in I, \forall j \in J, \forall k \in K, \forall l \in L)$

$$
\begin{aligned}
& g 1: X_{i}^{k} \leq A_{i}^{k} \cdot S_{i}^{k}\left(P_{i}^{k}\right) \\
& g 2: Z_{i}^{n} \leq \sum_{o \in O} Q_{o} \cdot d_{o, n, i}\left(P_{i}^{n}, P^{r}\right) \\
& g 3: Z^{r} \leq \sum_{o \in O} Q_{o} \cdot d_{o, r}\left(P_{i}^{n}, P^{r}\right) \\
& g 4: Z^{r} \leq \sum_{i \in I} \sum_{k \in K} X_{i}^{k} \\
& g 5: \sum_{i \in I} \sum_{k \in K}\left(e_{w}-e_{k}\right) \cdot X_{i}^{k}+\left(E_{n} \cdot Z^{r}-\left(\sum_{j \in J} e_{j}^{M} \cdot M_{j}+\sum_{l \in L} e_{l} \cdot Y_{l}+\sum_{j \in J} e_{j}^{N} \cdot N_{j}+e_{d} \cdot Z^{r}\right)\right) \leq \delta \\
& h 1: X_{i}^{k}+\sum_{l \in L} T_{j l} \cdot Y_{l}-M_{j}=0 \quad \forall j \text { corresponding to the end-of-life product } i \text { with } k \text { quality } \\
& h 2: N_{j}+\sum_{l \in L} T_{j l} \cdot Y_{l}-M_{j}=0 \quad \forall j \text { corresponding to a parts with external purchase availability } \\
& h 3: \sum_{l \in L} T_{j l} \cdot Y_{l}-M_{j}=0 \\
& h 4: \sum_{l \in L} T_{j l} \cdot Y_{l}-Z^{r}=0
\end{aligned} \begin{aligned}
& \forall j \text { corresponding to a parts without external purchase availability } \\
& h 5: N_{j}=0 \\
& h 6: M_{j}=0
\end{aligned}
$$$$
X_{i}^{k}, Y_{j}, Z_{i}^{n}, Z^{r}, M_{j}, N_{j}, P_{i}^{n}, P^{r}, P_{i}^{k} \geq 0(\forall i \in I, \forall j \in J, \forall k \in K, \forall l \in L)
$$

\section{CASE STUDY: SMARTPHONES}

\subsection{Scenario}

Smartphones, a representative example of short-life cycle products, are used as case studies to analyze the effects of generational commonality on the remanufacturing and manufacturing processes of products with short-life cycle. In the scenario, OEMs have produced only new smartphones so far, but they want to start producing remanufactured and new products from the new generation in order to respond to strengthened environmental laws and enhance the image of environmentally friendly brands.

OEMs need to collect EoL or returned products for remanufacturing, but it is difficult to secure enough materials for remanufacturing with new generation products. If the previous generation of products and new generations of products are designed to share some of the parts, the parts separated from the previous generation could be used for the remanufactured products of the new generation. It can increase the reusability of end-of-life products of previous generation and reduce associated costs, but at the same time it has the risk of reducing the appeal of a new generation of products by weakening the differentiation from previous generations. Given this trade-off of generational commonality, OEMs would like to 


\begin{tabular}{ll}
\hline Index & \\
\hline$I$ & Index set for model of product (old/new), $i \in I$ \\
$J$ & Index set for item (include part, product), $j \in J$ \\
$K$ & Index set for quality of end-of-life product, $\quad k \in K$ \\
$L$ & Index set for operation, $\quad l \in L$ \\
$O$ & Index set for market segment, $o \in O$ \\
\hline Design variable \\
\hline$X_{i}^{k}$ & Amount of take-back end-of-product $i$ with quality $k$ \\
$Y_{l}$ & Number of times operation 1 \\
$N_{j}$ & Amount of item $i$ to be purchased additionally \\
$M_{j}$ & Amount of item $i$ to be recycled \\
$Z_{i}^{n}$ & Production quantity of new product $i \in I$ \\
$Z^{r}$ & Production quantity of remanufactured product \\
\hline Parameter & \\
\hline$T_{j l}$ & Transition matrix \\
$A_{i}^{k}$ & Amount of end-of-life product $i$ availability with $k$ condition \\
$S_{i}^{k}$ & Take-back rate of end-of-life product $i$ with $k$ condition \\
$Q_{o}$ & Size of market segment \\
$d_{o, n, i}\left(P_{i}^{n}, P^{r}\right)$ & Market share of the new product $i$ in segment $o$ with selling price $\left(P_{i}^{n}, P^{r}\right)$ \\
$d_{o, r}\left(P_{i}^{n}, P^{r}\right)$ & Market share of the remanufactured product $i$ in segment $o$ with selling price $\left(P_{i}^{n}, P^{r}\right)$ \\
$C_{i}^{n}, E_{n}$ & Per-unit total cost for producing new product $i$ and environment impact for new product \\
$c_{j}^{N}, e_{j}^{N}$ & Per-unit cost and environment impact for purchasing new component, respectively \\
$c_{j}^{M}, e_{j}^{M}$ & Per-unit cost and environment impact for recycling, respectively \\
$c_{l}, e_{l}$ & Per-unit cost and environment impact for operating, respectively \\
$c_{d}, e_{d}$ & Per-unit cost and environment impact for marketing, respectively \\
$e_{k}$ & Per-unit environment impact of taking back an end-of-life product with quality $k$ \\
$e_{w}$ & Per-unit environment impact of an end-of-life product discarded by customer \\
$\delta$ & Target for the environment saving \\
\hline
\end{tabular}

explore the impact of the level of generational commonality on the manufacturing and remanufacturing processes and how it affects their profits.

To answer the aforementioned issue, this study compares the following scenarios:

- Baseline case (No-sharing): In this scenario, there is no shared part between the older and newer generations. The number of EoL products in the previous generation is larger than in the new generation but cannot be used in the process of producing new generation remanufactured products.

- Target case (Sharing): These are scenarios in which a certain portion of parts is shared between the old and the new generations. Because there are shared parts between the two generations, OEMs can use EoL products of old generation for the remanufactured products of new generation. Apply design scenarios that share parts between different generations to compare and analyze the results. The assumptions and input parameters used in this case study are extracted from various data sources. The types and parts prices of smartphones are assumed based on information on online component analysis sites (teardown.com). The reliability of the parts and the operation cost, recycling cost for remanufacturing are estimated based on previous studies (Kwak and Kim, 2017). For the new generation of products, it is assumed that they have approximately $15 \%$ higher reliability than for the previous product. Environmental impact parameters used values from Kwak and Kim (2017) using global warming potential (GWP).

\subsection{Assumptions on the supply of end-of-life products}

This model assumes that the quality of end-of-life products can be classified as good or poor, as in the previous study (Kwak and Kim, 2017). As shown in Table 2, it is assumed that there are more end-of-life products than the new generation because the previous generation was released to the market earlier. Use the response function used in the previous study to determine the number of products being taken back (Klausner and Hendrickson, 2000; Kwak and Kim, 2017). This function assumes that the takeback 
rates of end-of-life products increases linearly below a certain level of price, and that the it remains at 1 after critical prices. The critical prices are set differently depending on the generation and quality of smartphones.

Table 2. Parameters for end-of-life products

\begin{tabular}{llll}
\hline Product & Quality & Critical price & Availability \\
\hline Product 1 & Good & $\$ 80$ & 2000 \\
(Gen 1$)$ & Poor & $\$ 30$ & 4000 \\
\hline Product 2 & Good & $\$ 120$ & 1500 \\
(Gen 2) & Poor & $\$ 80$ & 500 \\
\hline
\end{tabular}

\subsection{Assumptions about new generation product design}

As shown in Table 3, several design alternatives for the new generation are assumed. This study considers 8 major components of smartphones. P1 and P2 represent old and new generation products, respectively. Each cell for P2 in Table 3 is marked as ' 1 ' if the same component is used within the older and newer generations (i.e., no upgrading) and ' 2 ' if different parts are used (i.e., upgrading).The baseline represents a no sharing case between two generations Case I, Case II, and Case III represent low sharing, intermediate and high sharing case, respectively.

Table 3. Product designs of new generation

\begin{tabular}{lccccc}
\hline \multirow{2}{*}{ Component } & P1 (Gen 1) & \multicolumn{5}{c}{ P2 (Gen 2) } \\
\cline { 3 - 6 } & & $\begin{array}{c}\text { Baseline } \\
\text { (No sharing) }\end{array}$ & $\begin{array}{c}\text { Case I } \\
\text { (Low) }\end{array}$ & $\begin{array}{c}\text { Case II } \\
\text { (Intermediate) }\end{array}$ & $\begin{array}{c}\text { Case III } \\
\text { (High) }\end{array}$ \\
\hline Display & 1 & 2 & 2 & 2 & 2 \\
Battery & 1 & 2 & 2 & 2 & 2 \\
Camera & 1 & 2 & 1 & 1 & 1 \\
Sensors & 1 & 2 & 2 & 2 & 2 \\
NAND & 1 & 2 & 2 & 1 & 1 \\
DRAM & 1 & 2 & 2 & 1 & 1 \\
Processor & 1 & 2 & 2 & 2 & 1 \\
BB+XCR & 1 & 2 & 2 & 2 & 1 \\
\hline
\end{tabular}

\subsection{Assumptions on demand model}

This model assumes that the market share of the target market segment is determined by the customer's utility of the performance, selling price and newness of the product. This study uses differences in component prices to demonstrate performance utility level because the performance difference felt by customers due to the sharing of components between generations should be presented. In general, if a part of the new generation is improved, the price of that part is higher than that of the previous generation. For example, when analyzing the price of parts for iPhone generation, it can be confirmed that the price of parts increased as performance of the camera, memory, display and others improved. In other words, if the price of the part has increased significantly compared to the previous generation, it means that the part has improved significantly. On the other hand, using the same parts of the previous generation without an upgrade tends to lower the price of the parts. This trend is used to reduce the customer's utility level of performance due to technological obsolescence if the same components are used between generations.

Equation 5 shows a utility function for the performance applied in this study. The $p_{i, \text { new }}, p_{i, \text { old }}$ represent the component $i$ 's price of new generation and the component $i$ 's price of old generation, respectively. This function assumes that the cost of the component determines the level of performance and that the customer's utility functions change depending on the level of performance. The performance level is 
calculated as a percentage of how much the price of parts of the next generation has increased compared to the previous generation.

$$
\operatorname{perf}_{\text {new }}=\sum\left(\frac{p_{i, \text { new }}}{\sum p_{i, \text { old }}}\right) \times \text { perf }_{\text {old }}
$$

The utility for the selling price is determined based on the customer segment by the critical price and current price information. As in the previous study, it is assumed that if the product price exceeds the critical price, it is not purchased (Kwak and Kim, 2017). The utility of the newness refers to the discount rate in the customer's perception that depends on whether the part of the product comes from a new component or from a used one.

Table 4 shows each of the competitors in the target market used in this model. Competitor 1 produces a new product that has high performance but high price. On the other hand, competitor 2 produces a low-priced new product, but its performance is low. Competitor 3 produces a high-performance product but sells them at relatively low prices due to remanufactured products.

Table 5 shows the target market segments. The market sizes are 5,000, 5,000, and 5,000, respectively. Market segment 1 is a group of performance-conscious customers with high critical prices, sensitive to product performance, and negative perception of remanufactured products. Market segment 3 is a group of price-sensitive customers that are relatively performance-sensitive and have a lower negative perception of remanufactured products than others. Market segment 2 prefers new products to segment 3 but prefer reasonably priced products to market segment 1 .

Equation 6 represents the multinomial logit model for demand. The customer's utility for the product depends on its performance, price and newness of product. For the utility of performance, it is assumed that different utility functions are applied because of the different sensitivity of each customer group and the relevant formulas are as shown in equation 6. $u_{s, o}$ is the utility of product $s$ in market segment $O$, where $S$ is the index set for choice set $(s \in S)$. This demand model is used in this paper, but can be changed to the demand model desired by the user.

$$
u_{\text {product }_{s, o}}=u_{s, \text { perf }, o} \times u_{s, \text { price }, o} \times u_{s, \text { new }, o}
$$

where

$$
\begin{aligned}
& u_{s, p e r f, o}= \begin{cases}\exp \left(\operatorname{perf}_{s}\right)^{2}, & \text { for the market segment } 1 \\
\exp \left(\operatorname{perf}_{s}\right), & \text { for the market segment } 2 \\
\sqrt{\exp \left(\operatorname{perf}_{s}\right),} & \text { for the market segment } 3\end{cases} \\
& u_{s, \text { price }, o}=\max \left(0,1-\frac{P_{s}}{\bar{P}_{o}}\right) \\
& u_{s, \text { new }, o}= \begin{cases}1, & \text { if choice } s \text { is a new product } \\
\beta_{o}, & \text { else choice } s \text { is a remanufactured product }\end{cases} \\
& d_{o, n, i}\left(P_{i}^{n}, P^{r}\right)=\frac{u_{o, n, i}}{\sum_{s \in S} u_{S, o}}, \quad d_{o, r}\left(P_{i}^{n}, P^{r}\right)=\frac{u_{o, r}}{\sum_{s \in S} u_{s, o}}
\end{aligned}
$$

Table 4. Parameters for competitors in the market

\begin{tabular}{llll}
\hline & Performance & Price & Newness \\
\hline Competitor 1 & 0.8 & $\$ 800$ & New \\
Competitor 2 & 0.5 & $\$ 500$ & New \\
Competitor 3 & 0.8 & $\$ 400$ & Remanufactured \\
\hline
\end{tabular}

Table 5. Targeted market segments

\begin{tabular}{llll}
\hline & Size & Critical Price $\left(\bar{P}_{l}\right)$ & $\begin{array}{l}\text { Utility discount factor for } \\
\text { remanufactured products }\left(\beta_{o}\right)\end{array}$ \\
\hline Segment 1 & 5000 units & $\$ 1000$ & 0.1 \\
Segment 2 & 5000 units & $\$ 800$ & 0.5 \\
Segment 3 & 5000 units & $\$ 600$ & 0.9 \\
\hline
\end{tabular}


Table 6. Optimization results (Profit)

\begin{tabular}{lllll}
\hline & $\begin{array}{c}\text { Baseline } \\
(\text { No sharing) }\end{array}$ & \multicolumn{1}{c}{$\begin{array}{c}\text { Case I } \\
(\text { Low })\end{array}$} & $\begin{array}{c}\text { Case II } \\
\text { (Intermediate) }\end{array}$ & \multicolumn{1}{c}{$\begin{array}{c}\text { Case III } \\
\text { (High) }\end{array}$} \\
\hline (1) Revenue (new) & $\$ 1,958,877$ & $\$ 1,836,706$ & $\$ 1,791,858$ & $\$ 1,078,487$ \\
(2) Cost (new) & $\$ 758,675$ & $\$ 686,012$ & $\$ 655,844$ & $\$ 549,555$ \\
(3) Profit (new) (=(1)-(2)) & $\$ 1,200,202$ & $\$ 1,150,694(-4 \%)$ & $\$ 1,136,014(-5 \%)$ & $\$ 1,078,487(-10 \%)$ \\
\hline (4) Revenue (reman.) & $\$ 1,078,819$ & $\$ 1,331,263$ & $\$ 1,318,563$ & $\$ 1,274,885$ \\
(5) Cost (reman.) & $\$ 787,276$ & $\$ 914,241$ & $\$ 920,254$ & $\$ 985,628$ \\
(6) Profit (reman.) $(=(4)-(5))$ & $\$ 787,276$ & $\$ 914,241(+16 \%)$ & $\$ 920,254(+17 \%)$ & $\$ 985,628(+25 \%)$ \\
\hline (7) Total profit $(=(3)+(6))$ & $\$ 1,987,478$ & $\$ 2,064,935(+4 \%)$ & $\$ 2,056,267(+3 \%)$ & $\$ 2,064,115(+4 \%)$ \\
\hline
\end{tabular}

\subsection{Optimization results}

The optimization results are compared to assess how the overall profit, production plans and pricing policies changed with the sharing of designs between generations. This section shows the optimal results of applying the assumptions and scenarios described above to the model.

Table 6 shows the total profits, including manufacturing and remanufacturing, for three different scenarios compared to the baseline. As shown in the no sharing and low sharing case, the less shared parts, the higher both the revenue and profit of the manufacturing sector compared to other cases. This is because the less shared parts between generations, the higher the performance and the higher the price compared to other cases. Sharing a small number of parts allows for high performance, which leads to increased profits. In case of remanufacturing, however, the less shared parts, the lower the profit of the remanufacturing sector, as shown in the Case III. This is because the profit is reduced because of increased costs for remanufacturing, although sharing fewer parts allows for high performance, which leads to increased revenue. Therefore, the overall profits are found to be almost equally high for Case I (Low sharing) and Case III (High sharing). Case I and Case III have the higher overall profits because they have the highest profits in the manufacturing sector and remanufacturing sector, respectively. The rate of increase/decrease in profit compared to the base case is shown in parentheses.

Table 7 shows the optimal buyback price, production quantities, and sales price in several scenarios. The results show that the less shared part, the higher the buyback price of the new generation product. This is because OEMs use the new generation of EoL/return products, which are relatively costly and rarely available in the market due to a reduction in the number of available parts in the previous generation. They set a higher price for buyback to collect the quantity needed for remanufacturing.

Table 8 represents the market share is changed according to different scenarios. As shown in the baseline case, there is a high market share in segments 1 and 2 with relatively high critical prices and performance importance. Compared with baseline and other cases, the market share decreases for relatively performance-sensitive customer groups, but increases for price-sensitive group.

\section{CONCLUSION}

OEMs who produce a line of new and remanufactured products should make the generational commonality decision when designing new generations of products. In this process, it is important to understand the way generational commonality affects the overall production process. To investigate the effect of the intergenerational commonality on the overall production process, the expanded mathematical model is

Table 7. Optimization results (Price, production quantity)

\begin{tabular}{llllll}
\hline & & Baseline & Case I & Case II & Case III \\
\hline Buyback price & Good & $\$ 0$ & $\$ 12$ & $\$ 16$ & $\$ 25$ \\
(Gen 1) & Poor & $\$ 0$ & $\$ 8$ & $\$ 8$ & $\$ 13$ \\
\hline Buyback price & Good & $\$ 120$ & $\$ 86$ & $\$ 81$ & $\$ 56$ \\
(Gen 2) & Poor & $\$ 80$ & $\$ 55$ & $\$ 50$ & $\$ 35$ \\
\hline \multirow{2}{*}{ Production quantity } & New & 3,090 & 2,877 & 2,824 & 2,624 \\
& Reman. & 2,000 & 2,813 & 2,812 & 2,838 \\
\hline \multirow{2}{*}{ Selling price } & New & $\$ 634$ & $\$ 638$ & $\$ 635$ & $\$ 620$ \\
& Reman. & $\$ 539$ & $\$ 473$ & $\$ 469$ & $\$ 449$ \\
\hline
\end{tabular}


Table 8. Optimization results (Market share)

\begin{tabular}{lllll}
\hline & & Segment 1 & Segment 2 & Segment 3 \\
\hline Baseline & New & $38 \%$ & $27 \%$ & $0 \%$ \\
(No sharing) & Reman. & $5 \%$ & $16 \%$ & $23 \%$ \\
\hline Case I & New & $37 \%(-1 \%)$ & $27 \%$ & $0 \%$ \\
(Low) & Reman. & $5 \%$ & $16 \%$ & $25 \%(+2 \%)$ \\
\hline Case II & New & $36 \%(-2 \%)$ & $27 \%$ & $0 \%$ \\
(Intermediate) & Reman. & $5 \%$ & $16 \%$ & $26 \%(+3 \%)$ \\
\hline Case III & New & $32 \%(-6 \%)$ & $26 \%(-1 \%)$ & $0 \%$ \\
(High) & Reman. & $5 \%$ & $15 \%$ & $28 \%(+5 \%)$ \\
\hline
\end{tabular}

used. Specifically, this model provides a detailed solution for the buyback, production, and sales if other generations of products are available in the process of remanufacturing.

The study results demonstrate that the commonality factor of the new and previous generations affects the profitability of overall production. When two generations of products are designed to have common components, the remanufacturing profits are greater than the baseline case product in which the components are not shared. At the same time, however, the profits from the manufacturing process of sharing case decrease compared to the baseline case. This is because sharing increases utilization rates of previous generations during remanufacturing, but at the same time, weakens differentiation from previous products in the manufacturing process. The results imply that profits do not increase or decrease monotonously depending on the level of component sharing.

Moreover, the results show that the commonality factor of the new and previous generations also affects the market share. Compared with baseline, the high sharing case is reduced the market share of performance-sensitive customer groups but increased the market share of price-sensitive customer groups.

These results show that there is an optimal generational commonality level that maximizes total profit while satisfying the environment impact saving. Through these analyses, the trade-off of the generational commonality can be identified on the entire production processes. The results provide significant insights into the sharing of components between generations.

\section{REFERENCES}

Aydin, R., Kwong, C.K. and Ji, P., 2015. “A novel methodology for simultaneous consideration of remanufactured and new products in product line design". International Journal of Production Economics, Vol. 169, pp. 127-140.

Aydin, R., Brown, A., Ali, A. and Badurdeen, F., 2017. “Assessment of End-of-life Product Lifecycle 'ilities"'. In IIE Annual Conference Proceedings, Institute of Industrial and Systems Engineers (IISE), (pp. 1691-1696).

Greenpeace reports. (2017) Guide to Greener Electronics [Online], Available at: from https:/www.greenpeace.org/usa/wp-content/uploads/2017/10/Guide-to-Greener-Electronics-2017.pdf

Hatcher, G.D., Ijomah, W.L. and Windmill, J.F.C., 2011. "Design for remanufacture: a literature review and future research needs". Journal of Cleaner Production, Vol. 19 No. 17-18, pp. 2004-2014.

Ijomah, W., 2002. A model-based definition of the generic remanufacturing business process.

Kim, S. and Moon, S.K., 2017. "Sustainable product family configuration based on a platform strategy". Journal of Engineering Design, Vol. 28 No. 10-12, pp. 731-764.

Klausner, M. and Hendrickson, C.T., 2000. "Reverse-logistics strategy for product take-back". Interfaces, Vol. 30 No. 3, pp. 156-165.

Kwak, M.J., Hong, Y.S. and Cho, N.W., 2009. "Eco-architecture analysis for end-of-life decision making". International Journal of Production Research, Vol. 47 No. 22, pp. 6233-6259.

Kwak, M. and Kim, H.M., 2011. "Assessing product family design from an end-of-life perspective". Engineering Optimization, Vol. 43 No. 3, pp. 233-255.

Kwak, M. and Kim, H., 2017. "Green profit maximization through integrated pricing and production planning for a line of new and remanufactured products". Journal of cleaner production, Vol. 142, pp. 3454-3470.

Kwak, M., 2018. "Optimal Line Design of New and Remanufactured Products: A Model for Maximum Profit and Market Share with Environmental Consideration”. Sustainability, Vol. 10 No. 11, p. 4283.

Simpson, T.W., Maier, J.R. and Mistree, F., 2001. "Product platform design: method and application". Research in engineering Design, Vol. 13 No. 1, pp. 2-22.

Thevenot, H.J. and Simpson, T.W., 2007. "A comprehensive metric for evaluating component commonality in a product family”. Journal of Engineering Design, Vol. 18 No. 6, pp. 577-598. 